\title{
Moisture Sorption as a Potential Condition Marker for Historic Silks: Noninvasive Determination by Near-Infrared Spectroscopy
}

\author{
XIAOMEI ZHANG and PAUL WYETH* \\ School of Archaeology and Museology, Peking University, Beijing 100871, P.R. China (X.Z.); and Textile Conservation Centre, University of \\ Southampton Winchester Campus, Park Avenue, Winchester, SO23 8DL, UK (P.W.)
}

\begin{abstract}
Given their ephemeral nature, the preservation of historic silks can be problematic. Rapid, on-site condition monitoring would offer significant benefits to conservators and museum curators concerned with continued access to collections. In this paper, near-infrared spectroscopy (NIR) is investigated as a noninvasive approach to the characterization of silk fabrics and particularly for determining the moisture content of silks as a potential age-related marker. Bands within the NIR spectrum of silk are assigned to contributions from water and the silk fibroin polymer. The water bands may be deconvolved to show separate contributions from bound and structural water. When silk is exposed to deuterium oxide, the water OH NIR bands are rapidly lost. The accompanying changes in the amide-related NIR absorptions reflect differential accessibility of regions within the semi-crystalline fibroin aggregate. NIR spectra were recorded while silk was maintained at a range of relative humidity; complementary gravimetry provided absolute reference data for moisture sorption. A single spectral parameter, the intensity of the water combination band, is sufficient to indicate the relative moisture content of silk and allows distinction of unaged and heat, light, and humidity aged silks. The results confirm that NIR has significant potential for on-site studies at collections in support of the preservation and access of our silk heritage.
\end{abstract}

Index Headings: Near-infrared spectroscopy; NIR spectroscopy; Deuterium exchange; Historic silk; Conservation; Aging; Condition; Moisture sorption.

\section{INTRODUCTION}

The origin of silk for textiles can be traced back over 5000 years to ancient China; the luxurious fabric was, of course, much sought after, and it was subsequently traded across the globe by way of the silk road, and eventually some was produced in other countries, including Europe. Historic silk fabrics are frequently part of museum collections all over the world. While the biopolymeric nature of the fiber produced by the silkworm (e.g., Bombyx mori) conveys some valuable properties such as strength, resilience, and luster, making silk ideal for textiles, it also leads to ready deterioration. Consequently silk artifacts may be somewhat fragile and problematic to handle, conserve, and display. Means to assess the condition of a fabric are then of particular value, informing conservation and curatorial decisions. Appropriate techniques have been developed that require just microsamples. ${ }^{1,2}$ However, rapid on-site, noninvasive monitoring would offer significant additional benefits.

In considering the variety of condition-related parameters that might be probed noninvasively, we have suggested moisture sorption as a potential candidate for natural textiles, with the water being quantified by diffuse reflectance nearinfrared spectroscopy (NIR). ${ }^{3}$ Portable near-infrared moisture meters are already widely used to determine the water content

Received 9 June 2006; accepted 20 November 2006

* Author to whom correspondence should be sent. Email: pw@soton.ac. uk. of a variety of agricultural and other products. ${ }^{4}$ More recently others have begun to transpose the NIR technique to the context of cultural heritage, e.g., using NIR to study archival paper $^{5-8}$ Here we present our results concerned with the development of its application to historic textiles and specifically aged silks.

Near-infrared spectroscopy has singular potential for interrogating the condition of organic artifacts, especially in association with univariate and multivariate spectral analysis. ${ }^{9,10}$ The radiation (typically within the range 12500-4000 $\mathrm{cm}^{-1}$ ) is efficiently transmitted through silica glass optical fibers, allowing remote interrogation using an appropriate probe. The effective sampling depth is of the order of $1 \mathrm{~mm}$ and is ideal for textiles. NIR absorptions arise from excitation of overtones and combinations of the mid-infrared fundamental vibrational modes. Due to their anharmonic nature, vibrations of $\mathrm{X}-\mathrm{H}$ bonds tend to dominate the NIR spectra, so for a proteinaceous silk fabric overtone and combination bands arising from $\mathrm{OH}$ (water, serine), $\mathrm{NH}$ (peptide) and $\mathrm{CH}$ (peptide, alanine, serine etc) containing moieties will be evident.

To investigate the potential of near-infrared spectroscopy for monitoring the moisture sorption of silk, samples of a modern Chinese silk fabric were first aged artificially. NIR spectra were then recorded while the silks were maintained at a range of relative humidity. Gravimetric analyses provided reference data for the unaged silk. To assist in band assignments and to further assess the kinetics of water exchange on silk, NIR spectra were also recorded during a deuterium-hydrogen exchange time course. The results presented below suggest that a single normalized spectral NIR parameter is sufficient to indicate the relative moisture content of silk and that moisture sorption is related to the condition of the silk.

\section{MATERIALS AND METHODS}

A commercially degummed, unweighted, and undyed Bombyx mori silk fabric was acquired in China. Fabric strips were subjected separately to extremes of heat, light, and humidity, effecting rapid degradation to an extent typical of historic textiles of a few hundred years old or more.

Thermal Aging. The fabric strips were placed in a laboratory convection oven at $125{ }^{\circ} \mathrm{C}$ for 20 days.

Light Aging. Samples were exposed to simulated sunlight in a Q-Sun Xenon Test Chamber model Xe1 for 12 days. The temperature was maintained at $40{ }^{\circ} \mathrm{C}$ during the light exposure, and the system was programmed to deliver $0.4 \mathrm{~W} / \mathrm{m}^{2}$ of light energy at $340 \mathrm{~nm}$. The positions of the samples were rotated regularly to ensure even exposure.

High Humidity Aging. Silk strips were put in $150 \mathrm{~cm}^{3}$ glass hybridization bottles with a tube containing $2 \mathrm{~cm}^{3}$ water, to provide $100 \%$ humidity. The bottles were sealed using a screwtop with an underlying PTFE-faced rubber septum and placed 


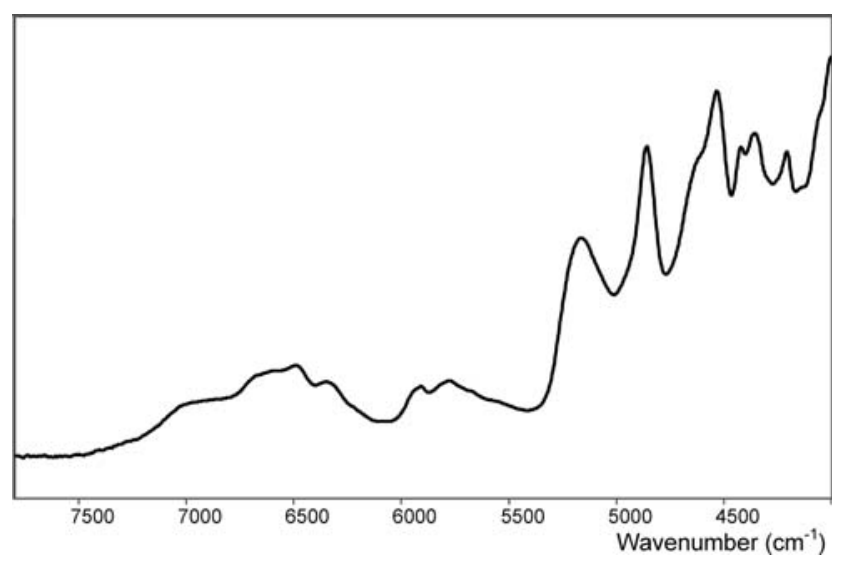

FIG. 1. The unprocessed NIR absorbance spectrum of Chinese silk (RH 45\%).

in a fan-assisted convection oven at $100{ }^{\circ} \mathrm{C}$. Aging was continued for 20 days.

Near-infrared diffuse reflectance spectra were recorded (in the absorbance mode) using a Perkin Elmer 'Spectrum One' FT-NIR spectrometer fitted with a near-infrared accessory (NIRA) having an inbuilt indium gallium arsenide (InGaAs) detector; spectra were captured over the range 7800-4000 $\mathrm{cm}^{-1}$, using 256 scans, and with a resolution of $8 \mathrm{~cm}^{-1}$. Spectra were subsequently manipulated with Galactic Industries GRAMS/32 AI (6.00) software. For spectral acquisition, samples were simply laid on the top of the sapphire window of the NIRA. Spectralon was used as a reflector for the background reference.

Deuterium exchange provides a convenient means of identifying moisture and other hydrogen exchangeable $\mathrm{O}-\mathrm{H}$ and $\mathrm{N}-\mathrm{H}$ related peaks in spectra. To follow hydrogen for deuterium exchange in the unaged and aged silks, a fabric sample was placed at the bottom of a glass vial, $1.0 \mathrm{~cm}^{3} \mathrm{D}_{2} \mathrm{O}$ in a small propylene tube was introduced, and the vial was capped. NIR spectra were then recorded periodically over 4 days.

To thoroughly assess the moisture sorption of the silks, aged and unaged samples were maintained under six different conditions of RH (relative humidity) $(0,25,34,45,64$, and $75 \%$ ) for two days, sufficiently long for equilibration, and NIR spectra were then recorded. To establish the appropriate humidity, a silk sample was placed in a capped glass vial containing a suitable saturated salt solution ${ }^{11}$ or phosphorus pentoxide powder held in a small polypropylene tube. Spectra were recorded through the capped glass vial. In each case, six replicates were prepared and four spectra of each were recorded, for statistical significance. It had already been established that the orientation of the silk did not affect the spectra.

The moisture contents of unaged silk samples maintained under the same RH conditions for the same length of time were also determined gravimetrically.

\section{RESULTS AND DISCUSSION}

Before commencing the detailed studies, to establish that NIR spectroscopy was an innocent procedure, a silk sample was left on the NIRA eight times longer than normal (2048 scans) and then aged at $125{ }^{\circ} \mathrm{C}$ for 10 days. There were no differences in the spectra for the NIR exposed and unexposed
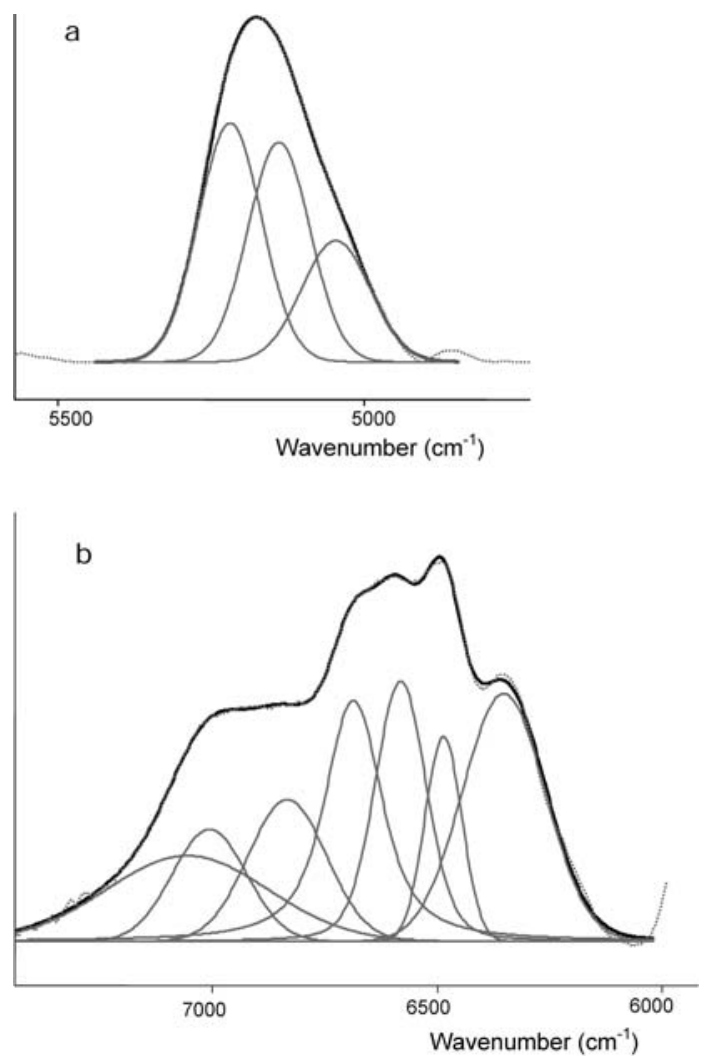

FIG. 2. The result of conservative deconvolution of (a) the water combination band, and (b) the spectral envelope at $6000-7500 \mathrm{~cm}^{-1}$. For deconvolution, the water combination band was isolated by spectral subtraction of silk held at $45 \%$ and $0 \% \mathrm{RH}$. The higher wavenumber spectral envelope is that from the $45 \%$ $\mathrm{RH}$ spectrum. In each case the spectrum is shown as a dotted line and the sum of the deconvolved peaks is overlaid as a solid line.

samples, both in the mid-infrared and near-infrared regions, either before or after thermal aging.

Near-Infrared Band Assignments. The NIR absorbance spectrum of unaged silk at intermediate RH is shown in Fig. 1. Water bands appear at $5170 \mathrm{~cm}^{-1}(\mathrm{O}-\mathrm{H}$ combination) and $7000 \mathrm{~cm}^{-1}$ (first overtone $\mathrm{O}-\mathrm{H}$ stretch). ${ }^{12}$ However, the combination band seems asymmetric (Fig. 2a) and can be deconvolved into at least three components $(5219,5139$, and $5046 \mathrm{~cm}^{-1}$ ). As the degree of hydrogen bonding to water increases, peaks are shifted to lower wave number. ${ }^{13}$ Water is generally considered to associate with natural fabrics in three ways: structural: a strongly associated monomolecular layer in the amorphous region that is only lost in extreme conditions; bound: absorbed moisture that fills the residual amorphous space and higher order pores and is lost at moderate relative humidities; and excess: the most loosely bonded water that fills interstices in the fabric and can be removed, for example, by centrifugation. ${ }^{3}$ While, at intermediate RH there will be little excess water, the three deconvolved peaks may represent bound and structural water. The two higher wavenumber bands perhaps offer a distinction of bound water with different degrees of hydrogen bonding, while the lowest wavenumber band might be assigned to strongly hydrogen bonded structural water, though there may be other interpretations. At the 25$75 \% \mathrm{RH}$ values employed in the moisture sorption study, structural water will be present throughout the range. While there may be little bound water at the lowest values, it is this 


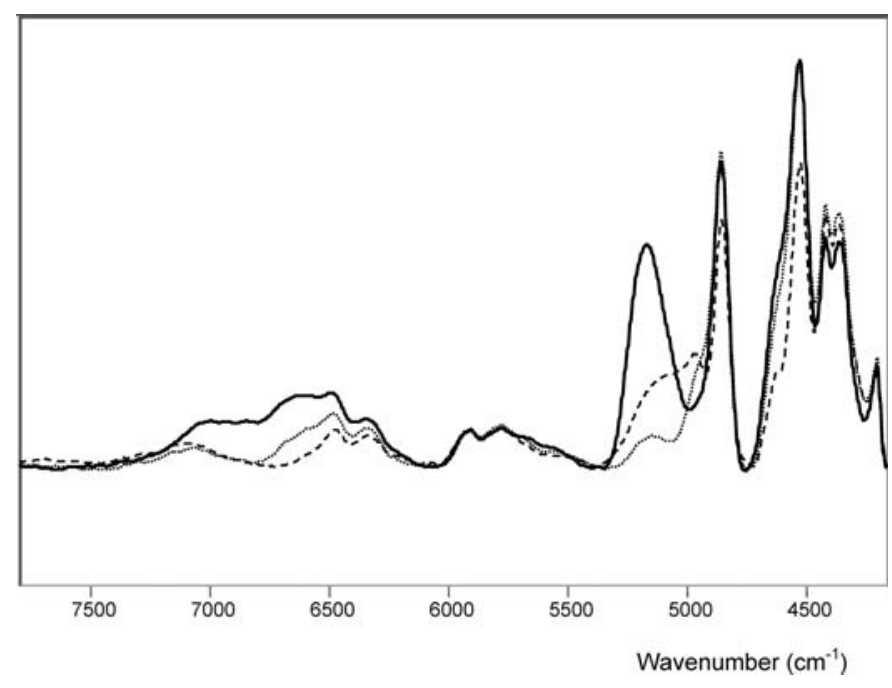

FIG. 3. NIR absorbance spectra recorded during the deuterium exchange time course for unaged silk at $0(-), 0.5(-)$, and $96(--)$ h. The spectra have been offset and baseline corrected to allow easier visualization of the changes. As observed, no change is expected in the methylene overtone region (5900$5700 \mathrm{~cm}^{-1}$ ).

component that will increase with rising $\mathrm{RH}$, as represented by the observed growing intensity of the $5170 \mathrm{~cm}^{-1}$ envelope.

The water first overtone is concealed in the spectral envelope between 6000 and $7500 \mathrm{~cm}^{-1}$ (Fig. 2b). There appear to be just two distinct bands at higher wavenumber $\left(7006,6833 \mathrm{~cm}^{-1}\right)$ that could be assigned to the water, though of course it would be possible to include an extra band in the deconvolution to match the number found in that for the combination band. The broad highest wavenumber band $\left(7080 \mathrm{~cm}^{-1}\right)$ may be due to the $\mathrm{OH}$ of serine, which is in relatively high abundance in fibroin. This peak is evident in the silk spectrum at $0 \% \mathrm{RH}$ and is not significantly affected during deuterium exchange, as would be expected since the serine is a constituent of the crystalline regions of silk fibroin. The remaining bands under the envelope also appear in the spectrum at $0 \% \mathrm{RH}$ and are presumably $\mathrm{N}-\mathrm{H}$ related. As silk is a semi-crystalline aggregate of the protein fibroin, multiple amide $\mathrm{N}-\mathrm{H}$ contributions are anticipated from the amorphous regions and the more strongly hydrogen bonded crystalline zones. Besides the understanding that stronger hydrogen bonding results in a shift to lower wavenumber, deuterium exchange is also valuable in distinguishing these contributions, as it will proceed more rapidly in the amorphous parts of polymer aggregates. ${ }^{14,15}$ Then, together with a comparison of the assignments for polymeric-amiderelated bands given by others, ${ }^{16-18}$ this leads us to tentatively suggest the following assignments for silk: $6686 \mathrm{~cm}^{-1}$ first overtone amide A v(NH) amorphous; $6581 \mathrm{~cm}^{-1}$ amide A $v(\mathrm{NH})$ amorphous + first overtone $v\left(\right.$ amide II); $6486 \mathrm{~cm}^{-1}$ first overtone amide A $v(\mathrm{NH})$ crystalline; and $6352 \mathrm{~cm}^{-1}$ amide A $v(\mathrm{NH})$ crystalline + first overtone $v$ (amide II). (The significance of the particular vibrational modes is not important here, but is described elsewhere. ${ }^{19}$ )

In a similar way, other regions in the spectra are assigned as follows: 5900-5700 $\mathrm{cm}^{-1}$ first overtone $v(\mathrm{CH})$; 5000-4700 $\mathrm{cm}^{-1}$ amide A $v(\mathrm{NH})+$ amide I/amide II; $4700-4500 \mathrm{~cm}^{-1}$ amide A $v(\mathrm{NH})+$ amide III/amide B + amide II; and 4500$4000 \mathrm{~cm}^{-1} \mathrm{v}+\delta(\mathrm{CH})$. The last of these regions is typical for a

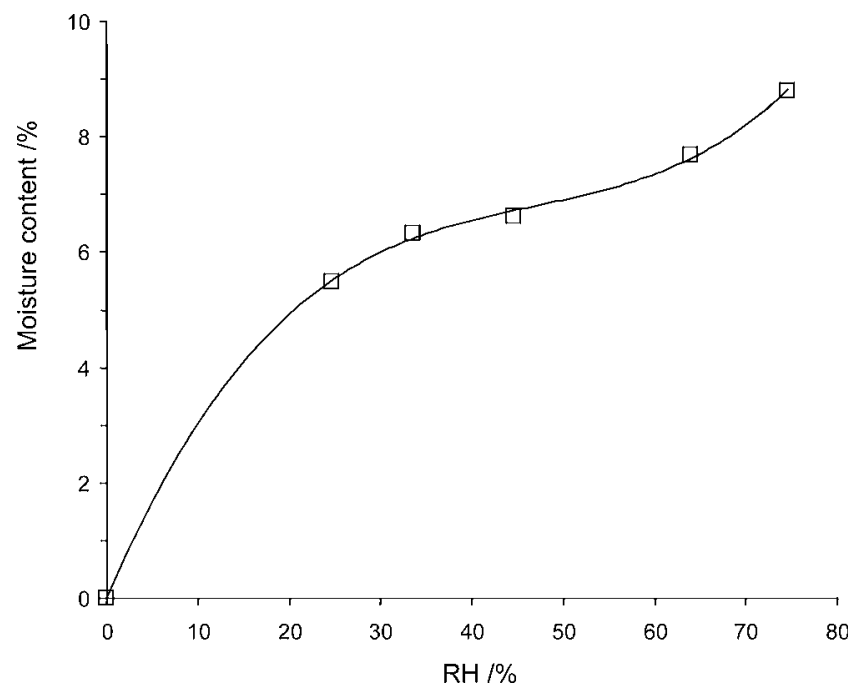

FIG. 4. The sorption isotherm for Bombyx mori silk $\left(20^{\circ} \mathrm{C}\right)$ determined by gravimetry. The size of the symbols is indicative of the standard error at each data point. The line is simply presented for easier visualization of the trend; it was generated automatically in MS Excel using a third-order polynomial and is not fitted to a particular model.

particular protein, being dependent on the amino acid composition. $^{20}$

Kinetics of Deuterium Exchange. As suggested above, the deuterium exchange experiments highlight those $\mathrm{OH}$ and $\mathrm{NH}$ NIR bands that arise from water and the accessible regions of silk fibroin. Both the water $\mathrm{OH}$ combination (envelope at 5170 $\mathrm{cm}^{-1}$ ) and overtone (7006, $6833 \mathrm{~cm}^{-1}$ ) bands very rapidly decline in intensity (Fig. 3). The amorphous amide NH bands $\left(6686,6581,4620 \mathrm{~cm}^{-1}\right)$ show evidence of slightly slower but still rapid exchange, while the bands assigned to the more strongly hydrogen bonded $\mathrm{OH}$ and $\mathrm{NH}$ moieties in the crystalline zones $\left(7080,6486,6352,4859,4529 \mathrm{~cm}^{-1}\right)$ reduce in intensity much more slowly. The new bands appearing over the exchange time course (e.g., $5150 \mathrm{~cm}^{-1}$ and $4970 \mathrm{~cm}^{-1}$, which are superimposed close to the original water combination and amide $\mathrm{A} v(\mathrm{NH})$ crystalline + amide I combination bands, respectively) must be OD and ND related. ${ }^{15,16}$ The data for the aged silks is very similar, though for the heat-aged sample alone exchange on amide in the amorphous region was inhibited slightly, perhaps suggesting a degree of cross-linking.

Moisture Sorption: Gravimetry. The moisture content of unaged silk at the different RH values is shown in Fig. 4. Clearly, silk is hygroscopic and exhibits typical behavior for a natural fiber consisting of a semi-crystalline hydrophilic polymer aggregate.

Moisture Sorption: Near-Infrared Spectroscopy. Secondary parameter correlations with NIR spectra often involve multivariate analysis. ${ }^{9,10}$ However, in the case of water there are very distinct bands in the NIR spectra, allowing a simpler univariate approach, coupled, as appropriate, with spectral preprocessing and normalization. The spectra of the silks held at the variety of $\mathrm{RH}$ values were first analyzed using this simpler approach.

Comparative moisture sorption was determined from the relative intensity of the prominent water combination envelope at $5170 \mathrm{~cm}^{-1}$. Kubelka-Munk transformation of the spectra followed by application of a 19 point gap second derivative and normalization to the methylene first overtone band was found 


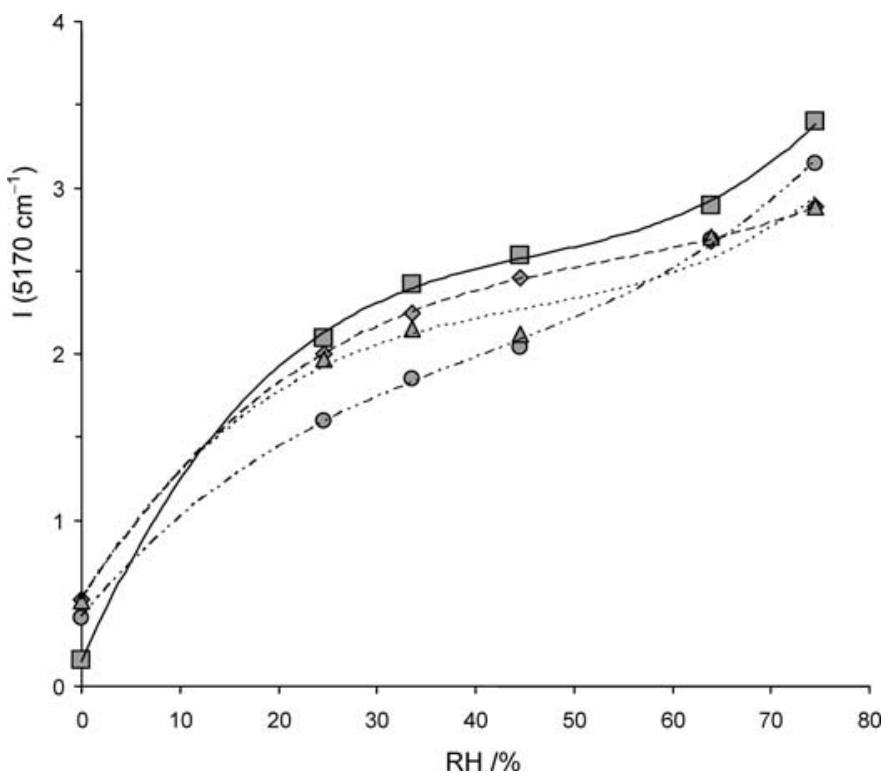

FIG. 5. Plots of the normalized intensities of the water combination bands, $I$ $\left(5170 \mathrm{~cm}^{-1}\right)$, for the unaged $(-\square-)$ and aged (heat $---\Delta---;$ light $--\downarrow--$, humidity - - - - - - ) silks. The size of the symbols is indicative of the standard error amongst the set of 24 replicate spectra at each data point. Thirdorder polynomial trendlines are again included to aid visualization.

to best improve the quality of the data. The Kubelka-Munk transform removes some spectral nonlinearity for the univariate case, second-degree derivatization serves to remove offset and sloping baseline differences, in particular eliminating some of the effects of differential scattering, and normalization may further moderate the influence of scattering besides accounting for sample thickness variations.

The NIR-derived relative moisture sorption profiles for the unaged and aged silks, based on the intensity of the water combination band $\left(5170 \mathrm{~cm}^{-1}\right)$, are illustrated in Fig. 5; the match with the unaged silk gravimetric profile is clear. Indeed, as illustrated for the unaged sample in Fig. 6, there is a linear correlation with moisture content, which includes both the structural and increasing bound components, and absolute quantitation of moisture is possible. However, the normalized intensity of the water combination band alone could suffice as a condition-related marker. There is a reasonably clear distinction for the aged samples that show lower values for the relative intensity, suggesting reduced sorption.

Incidentally, full spectral partial least squares regression analysis was also applied to the unaged sample set and a reasonable correlation was obtained, but in this case it offered no increased confidence over the simpler univariate approach.

\section{CONCLUSION}

Near-infrared spectroscopy offers a noninvasive approach to the characterization of silk fabrics and a simple method for determining the relative moisture content of silks.

Bands within the NIR spectrum of silk can be assigned to contributions from water (both bound and structural) and amide moieties within the amorphous and crystalline zones of the semi-crystalline silk fibroin aggregate. When silk is exposed to an atmosphere containing $\mathrm{D}_{2} \mathrm{O}$, the water $\mathrm{OH}$ bands are rapidly lost, but the NIR spectra show differential exchange of the

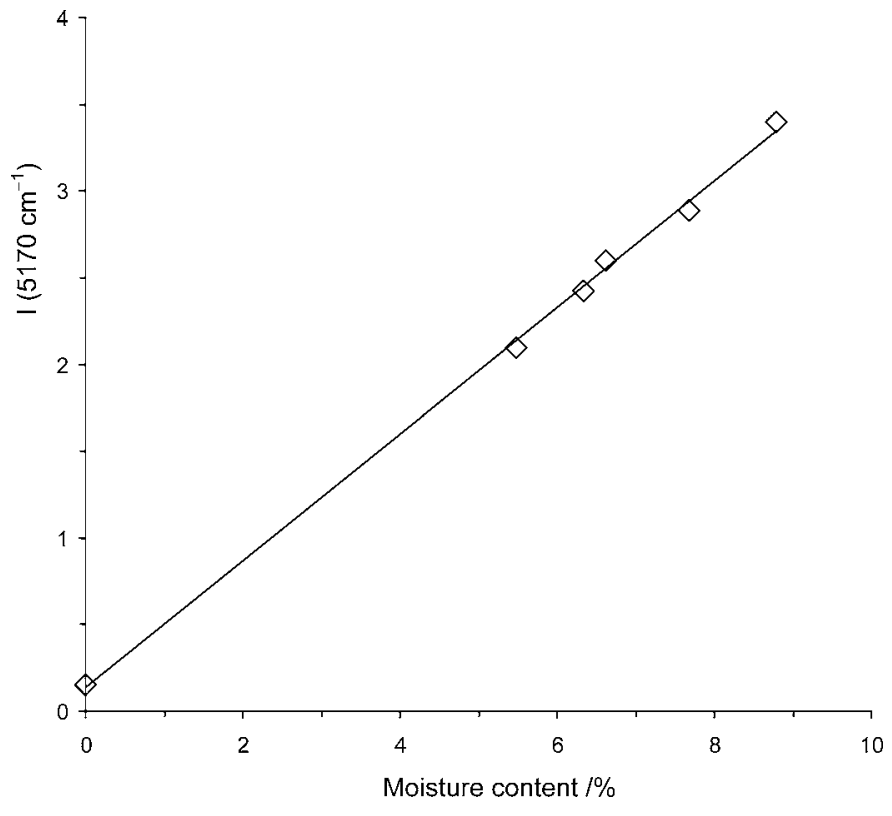

FIG. 6. Linear correlation of the normalized water combination band intensity, $I\left(5170 \mathrm{~cm}^{-1}\right)$, and the absolute moisture content of silk (correlation coefficient 0.99 ; standard deviation in $I, 0.049$ ). The size of the symbols is indicative of the standard errors. The line is the result of least squares linear regression.

amide $\mathrm{NH}$ and reflect the relative accessibility of the amorphous regions.

A single normalized NIR spectral parameter, the intensity of the water combination envelope at $5170 \mathrm{~cm}^{-1}$, is sufficient to indicate the relative moisture content of silk. This could offer a means to real-time monitoring of humidification, for example, a frequent preliminary procedure used by textile conservators to imbue a deteriorated historic fabric with enhanced pliability. The NIR spectral parameter provides distinct profiles for unaged and heat, light, and humidity aged silks. The results confirm that moisture sorption is related to the condition of silks and, further, that NIR spectroscopy has significant potential for on-site studies in support of the preservation and access of our silk heritage. However, since for historic artifacts conservation ethics will limit interrogation to ambient conditions, precluding the determination of a sorption profile, a more complex analysis may be required. In a subsequent communication we will report on the more involved multivariate spectral data analysis, which encouragingly appears to find predictive correlations with primary condition parameters, such as tensile strength.

\section{ACKNOWLEDGMENTS}

This research was completed while X.Z. was on study leave as a Research Fellow in the AHRC Research Centre for Textile Conservation and Textile Studies, at the Textile Conservation Centre and P.W. was on AHRC supported study leave. We are indebted to Maria Hayward, Director of the Research Centre, and Nell Hoare, Director of the TCC. We are also pleased to acknowledge additional funding from the British Academy. Henryk Herman, Actinic Technology, kindly helped us with the multivariate spectral analysis. This work would not have been possible without the support of all our colleagues at the TCC.

1. P. Garside and P. Wyeth, Appl. Spectrosc. 59, 1242 (2005).

2. K. Hallett and D. Howell, "Size Exclusion Chromatography as a Tool for Monitoring Silk Degradation in Historic Tapestries", in First annual conference of the AHRC Research Centre for Textile Conservation and Textile Studies, Scientific Analysis of Ancient and Historic Textiles: 
Informing Preservation, Display and Interpretation, Winchester, 13-15 July 2004, R. Janaway and P. Wyeth, Eds. (Archetype Publications, London, 2005), p. 143.

3. P. Wyeth, "Signatures of Aging: Correlations with Behaviour", in First annual conference of the AHRC Research Centre for Textile Conservation and Textile Studies, Scientific Analysis of Ancient and Historic Textiles: Informing Preservation, Display and Interpretation, Winchester, 13-15 July 2004, R. Janaway and P. Wyeth, Eds. (Archetype Publications, London, 2005), p. 137.

4. J. S. Shenk, J. J. Workman Jr., and M. O. Westerhaus, "Application of NIR Spectroscopy to Agricultural Products", in Handbook of Near-Infrared Analysis, D. A. Burns and E. W. Ciurczak, Eds. (Marcel Dekker, New York, 2001), 2nd ed., Chap. 16, p. 419.

5. M. Anders, P. Behnsen, and P. Plew, "Paper Chemistry: Development of a New Method for Non-destructive Analysis of Paper", in Preprints X IADA Congress, Göttingen, Germany, 22-26 September 2003 (IADA, OnlinePublisher: Wolfgang Jaworek, http://palimpsest.stanford.edu/iada/ta03_t. html, 2003) p. 11.

6. H. Yonenobu, S. Tsuchikawa, and H. Oda, J. Near Infrared Spectrosc. 11, 407 (2003).

7. D. Lichtblau and M. Anders, "Characterization of paper by near infrared spectroscopy, 28-30", in Proceedings of the International Conference Durability of paper and writing, November 16-19, 2004, Ljubljana, Slovenia, J. Kolar, M. Strlic, and J. B. G. A. Havermans, Eds. (National and University Library, Ljubljana, Slovenia, 2004), ISBN 961-6162-98-5.

8. http://www.science4heritage.org/survenir 05-06-2006.

9. H. M. Heise and R. Winzen, "Fundamental Chemometric Methods", in Near-Infrared Spectroscopy: Principles, Instruments, Applications, H. W.
Siesler, Y. Ozaki, S. Kawata, and H. M. Heise, Eds. (Wiley-VCH, Weinheim, Germany, 2002), Chap. 7, p. 125.

10. E. Cleve, E. Bach, and E. Schollmeyer, Anal. Chim. Acta 420, 163 (2000).

11. L. N. Bell and T. P. Labuza, Practical Aspects of Moisture Sorption Isotherm Measurement and Use (AACC Egan Press, Egan, MN, 2000), 2nd ed.

12. L. Bokobza, "Origin of Near-Infrared Absorption Bands", in NearInfrared Spectroscopy: Principles, Instruments, Applications, H. W. Siesler, Y. Ozaki, S. Kawata, and H. M. Heise, Eds. (Wiley-VCH, Weinheim, Germany, 2002),Chap. 2, p. 11.

13. C. E. Miller, "Chemical Principles of Near-Infrared Technology", in NearInfrared Technology in the Agricultural and Food Industries, P. Williams and K. Norris, Eds. (American Association of Cereal Chemists, Inc., St. Paul, MN, 2001), Chap. 2, p. 19.

14. P. Lagant, G. Vergoten, G. Fleury, and M. H. Loucheux-Lefebvre, Eur. J. Biochem. 139, 137 (1984).

15. S. Tsuchikawa, H. Yonenobu, and H. W. Siesler, Analyst (Cambridge, U.K.) 130, 379 (2005)

16. P. Wu and H. W. Siesler, Macromol. Symp. 143, 323 (1999).

17. M. Miyazawa and M. Sonoyama, J. Near Infrared Spectrosc. 6, A253 (1998).

18. N. Chaupart and G. Serpe, J. Near Infrared Spectrosc. 6, 307 (1998).

19. H. G. M. Edwards and P. Wyeth, "Ancient Textile Fibers", in Raman Spectroscopy in Art and Archaeology, H. G. M. Edwards and J. M. Chalmers, Eds. (Royal Society of Chemistry, Cambridge, 2005), Chap. 19, p. 304.

20. J. Wang, M. G. Sowa, M. K. Ahmed, and H. H. Mantsch, J. Phys. Chem. 98, 4748 (1994). 